\title{
Broadband continuously tunable all-fiber laser based on OPG for CARS imaging
}

\author{
I. Aporta, M. A. Quintela, J. M. López-Higuera
}

\begin{abstract}
A broadband continuously tunable SESAM all-fiber laser based on optical parametric generation (OPG) for Coherent anti-Stokes Raman Scattering (CARS) imaging applications is presented in this article. This laser structure is built on allpolarization maintaining fibers yielding high environmental stability. The OPG is based on PM photonic crystal fiber as parametric gain pumped by a wavelength-tunable $(1025 \mathrm{~nm}$ to $1055 \mathrm{~nm}$ ) and repetition rate-tunable $(3 \mathrm{MHz}$ to $500 \mathrm{KHz}) \mathrm{Yb}$ doped pulsed fiber laser. The all-fiber structure features free polarization adjustment in slow-axis tunable operation with a wavelength range from 770 to $940 \mathrm{~nm}$ for signal radiation and 1225 to $1510 \mathrm{~nm}$ for idler radiation. The pump and signal pulses (CARS-Stokes and CARS-pump respectively) are intrinsically overlapped, both spatially and temporarily, due to the four-wave mixing (FWM) generation and emitted from a single fiber end. The CARS-Stokes and CARS-pump pulse duration vary between 25 to 32 ps and 21 to 24 ps respectively in the whole band, ensuring a good overlap of the generated signals, and both exhibit a Gaussian profile with high spatial beam quality. The frequency conversion allows to obtain a frequency difference between the generated signals from 1100 to $3300 \mathrm{~cm}^{-1}$. The reported low-cost laser source is ideal for bio-imaging applications, especially in CARS imaging, due to its compactness, simplicity, robustness and ease of handling. A patent has been filed based on this technology.
\end{abstract}

Index Terms-Optical fiber lasers, Mode locked lasers, Laser tuning, Fiber nonlinear optics.

\section{INTRODUCTION}

Is $\mathrm{N}$ the last decades, the development of coherent radiation sources allowing emission wavelengths which conventional lasers could not provide, has been widely investigated. This manner, wavelength-based light conversion techniques have been a popular method to achieve the generation of light in wavelength ranges where active materials do not provide the necessary gain. Light sources based on these techniques have attracted a lot of interest in sensing applications [1,2] and biomedical imaging [3-5]. For this reason, lasers based on optical parametric interactions are well-suited to fulfill this purpose due to its capability of being continuously tunable over a broad range, high conversion efficiency and large frequency shifts [6, 7]. Optical parametric oscillators (OPOs) have been developed throughout years being the most commonly option utilized in these applications, but these type of laser structures

Manuscript received. This work was supported by the Spanish Comisión Interministerial de Ciencia y Tecnología within project TEC2016-76021-C2-2$\mathrm{R}$ (AEI/FEDER, UE) and project PID2019-107270RB-C21/ AEI / $10.13039 / 501100011033$.

I. Aporta is with the Photonics Engineering Group and CIBER-bbn, Universidad de Cantabria, 39005 Santander, Spain (e-mail: aportai@unican.es) are very expensive, bulky and need costly adjustment and maintenance. Over the past few years, fiber lasers based on OPG have become a promising solution because of the all-fiber format which can provide similar wavelength tunability in alignment-free operation with high environmental stability [8, 9]. Whereas OPOs are clearly not compatible with fiber integrated systems, fiber lasers based on OPG are designed to solve this problem being a cost-effective option with compactness and robustness [10, 11]. Furthermore, these oscillators have interesting advantages as: good beam quality, high power stability and the capability of reach hundreds of nanometers ensuring a tunable broadband range [7, 12-14]. The OPG is based on degenerate four-wave mixing (FWM) in a highly nonlinear fiber, namely photonic crystal fibers (PCF) $[15,16]$. The non-linear parametric interaction is described by the third-order nonlinearity $\chi_{3}$ and owing to this nonlinearity, two new waves named signal and idler photons are generated from two pump photons during the optical frequency conversion process in the fiber [17]. The frequencies of the generated photons are determined by the phase-matching condition which depends on the peak power and wavelength of the seed laser used to pump the optical fiber used as parametric gain and the dispersion profile of this optical fiber [18].

On another hand, passively SESAM-based mode-locked (ML) ytterbium fiber lasers are well known as reliable tools to achieve environmentally maintenance-free stable all-fiber pulsed lasers with compact size, wavelength-tunable capability, picosecond pulse duration and spectrally narrow[19-21]. In addition, modelocked YDF-based fiber lasers have focused on all-normal dispersion cavities to perform fiber integrated setups with no need of dispersion compensation [22, 23]. These requirements are essential for fundamental researches, such as optical microscopy [24], frequency metrology [25]... especially in biophotonics [26] and non-invasive label-free imaging techniques as: optical coherence tomography (OCT) [27], multiphoton microscopy (MPM) [28, 29] and coherent anti-Stokes Raman scattering (CARS) [30-32]. In particular, CARS microscopy has been demonstrated as a potent technique in biomedical applications providing high sensitivity and video-rate and image-rate speed using ultrashort laser sources [33]. This microscopy technique allows in-vivo imaging of living cells at real time, obtaining chemically selective contrast imaging without the use of any labels by probing the intrinsic vibrational resonance of molecular bonds. As it has been mentioned, CARS

M. A. Quintela and J. M. Lopez-Higuera are with the Photonics Engineering Group, CIBER-bbn and IDIVAL, Universidad de Cantabria, 39005 Santander, Spain (e-mail: angeles.quintela@unican.es; miguel.lopezhiguera@unican.es) 
is a FWM process and the generated pulsed signals require to be synchronized at different wavelengths. This is the critical point for CARS as it strongly depends on the fiber based generation of a synchronized pulse train at a second wavelength. Over the past few years, OPG by FWM in PCF has been demonstrated to be the best option to generate pump and stokes pulses passively synchronized for CARS [30, 34]. Moreover, the use of long-picosecond pulses with narrow bandwidths in an integrated all-fiber structure is ideal and can be applied directly to CARS microscopy [35]. Indeed, the narrow spectral widths allow a high spectral resolution with minimum parasitic distortion of the pulses due to dispersion, and the tens of ps pulses enable spatially and temporary overlapped components generated in the frequency conversion through FWM in PCF [3].

In this paper, a new experimental implementation of a compact, low-cost, free-alignment broadband continuously tunable allfiber based on OPG for CARS imaging application has been reported. The all-fiber system comprises a spectrally filtered fiber master oscillator power amplifier (MOPA), which is tunable in wavelength-emission and repetition-rate, and a parametric frequency conversion stage based on FWM in PCF. The whole setup is built out of PM commercial fiber components making a cost-efficient laser structure with high environmental stability. The picosecond wavelength-tunable master oscillator (seed laser) is based on Ytterbium doped fiber (YDF) as the active gain medium, an in-fiber band-pass filter acting as the tunability device and placed on a fiber loop mirror (FLM) at one extreme of the cavity and a fiber-coupled SESAM as the saturable absorber mirror to perform the mode-locking. This seed laser delivers a pulse train in the range of $1020 \mathrm{~nm}$ to $1060 \mathrm{~nm}$ with a spectral width of $80 \mathrm{pm}$, a repetition rate of 3 $\mathrm{MHz}$ and a pulse duration between 26 and $32 \mathrm{ps}$ depending on the pump level. The construction of the YDF amplifiers with PM single-mode fiber components allows for a compact fiber structure owing to the reduced average power requirements caused by the low repetition rate. Besides, the inclusion of a pulse picker as a repetition-rate selector of the fiber laser permits a variation in the range of $3 \mathrm{MHz}$ to $500 \mathrm{KHz}$ yielding a peak power of more than $5 \mathrm{~kW}$ in all the emission band. The OPG based on FWM in PCF delivers synchronized components (generated signal pulses used as CARS pump and the residual fundamental pump pulses serving as CARS Stokes) from a single fiber end for the whole working range of the FOPO with pulse durations between 21 to 24 ps and 26 to 32 ps respectively. The generated signal bandwidths work in the range of 10 to $35 \mathrm{~cm}^{-1}$ and the frequency shifts between this component and the residual fundamental pump laser pulses allow to access resonance from 1100 to $3300 \mathrm{~cm}^{-1}$.

\section{EXPERIMENTAL SETUP AND PRINCIPLE}

The schematic diagram of the proposed experimental setup is shown in Fig. 1. As is depicted in Fig.1 the all-fiber structure is based on a passively ML tunable seed laser, a two-stage YDF amplifier and a OPG based on FWM in PCF. In this experimental setup, the OPG stage is pumped by the amplified YDF seed laser. The seed laser is composed by an in-fiber bandpass filter (FWHM bandwidth of $100 \mathrm{pm}$ and resolution of $0.02 \mathrm{~nm}$ ) placed on a FLM composed by a wavelength-sensitive circulator, a delay-line of 32 meters of PM fiber (Nufern 980XP), a $90 \mathrm{~cm}$-long YDF (Nufern PM-YSF-LO) as the active gain medium, an in-line broadband slow-axis polarizer and a SESAM (Batop $\mathrm{GmbH}$ ) as the ML device with a modulation depth of $29 \%$, a relaxation time of $9 \mathrm{ps}$ and a saturation fluence of $100 \mu \mathrm{J} / \mathrm{cm}^{2}$. The seed laser output is achieved through a 90:10 optical coupler using the 10\% port. The YDF is pumped by a $976 \mathrm{~nm}$ laser diode (LD) of $750 \mathrm{~mW}$ via the $20 \%$ port of an 80:20 optical coupler through a wavelength division multiplexer (WDM). The pre and main-amplifiers are composed of 1.2 and 2.3 meters-long respectively of YDF with a $250 \mathrm{~dB} / \mathrm{m}$ absorption (Nufern PM-YSF-HI). The preamplifier is pumped by the $80 \%$ port of the optical coupler spliced to the LD used to pump the seed laser. The main amplifier is pumped by a $976 \mathrm{~nm}$ LD with an output power of $750 \mathrm{~mW}$. Both stages are isolated to protect the whole fiber structure and the seed laser from possible back reflections. A pulse picker (G\&H FCAOM) is set after the pre-amplifier to select the repetition rate of the ML seed laser which has a wavelength range of 1020 to $1060 \mathrm{~nm}$. The OPG stage is composed by a 1.3 meters-long PCF (NKT Photonics LMA-5PM) and a pigtailed collimator to focus the output beam of the fiber laser system.

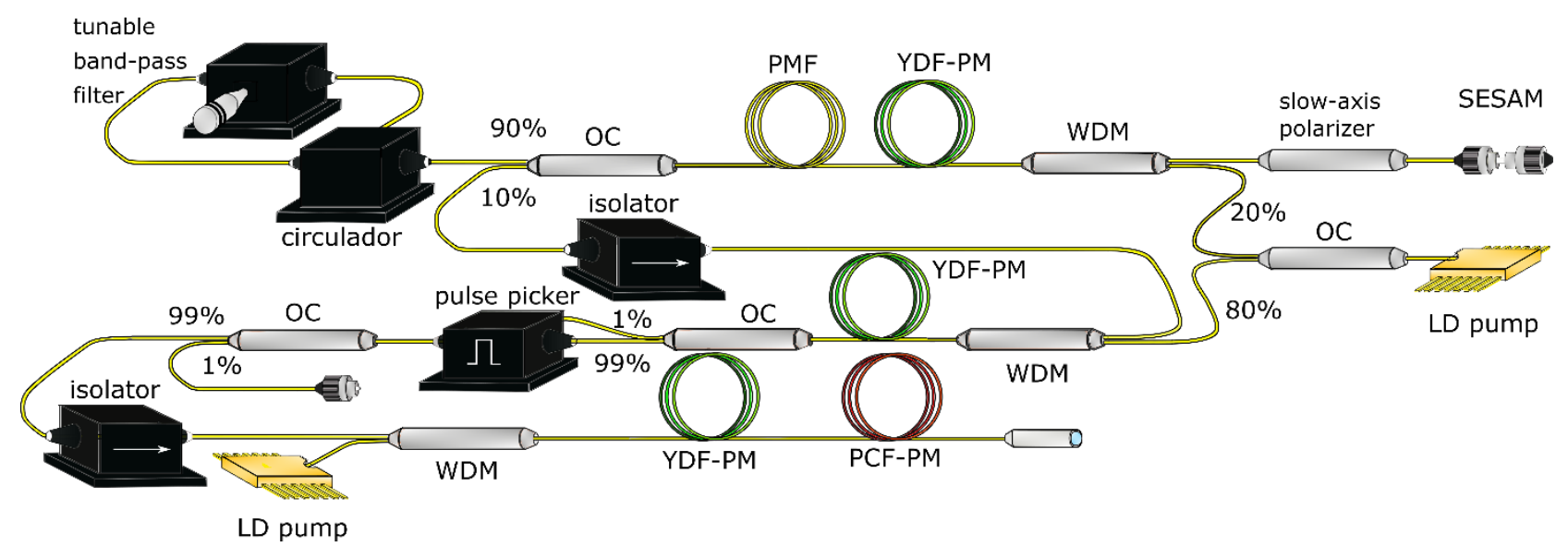

Fig.1 Schematic diagram of the experimental setup. 
The MOPA system generates a spectrally filtered wavelengthtunable and repetition-rate tunable pulse train which is amplified by a two-stage amplifier reaching the necessary peak power to perform the optical parametric frequency conversion by degenerate FWM in PCF. The frequency difference between the signal generated in this process and the fundamental laser that pumps the OPG stage is determined by the pump emission wavelength and the PCF dispersion profile, which defines the phase-matching condition of the parametric generation. The frequency shift between these components can be changed by tuning the emission wavelength of the pump fiber laser that drives the FWM process covering the resonance from 1100 to $3300 \mathrm{~cm}^{-1}$. As it has been explained, the synchronization of the pump pulses and the signal pulses is inherent to the FWM process, and both components are overlapped at the fiber output (spatially and temporary). In fact, these synchronized train of pulses are available to be used in a CARS microscope with no need of inserting delay lines or combining volume optics that would increment the complexity of the overall system. This compact and reliable fiber laser system is a low-cost solution and ease of handling, where the generated signals are emitted through a collimated fiber output for in-vivo imaging [36].

\section{RESULTS AND DISCUSSION.}

In this experimental configuration, stable self-started ML pulse train with continuously tunable wavelength has been achieved in the seed laser. Fig.2 shows the spectral, temporal and radio frequency characterization of the seed laser emitting at 1042.5 $\mathrm{nm}$ at a launched pump power of $78 \mathrm{~mW}$ (stable single-pulse operation). As is depicted in Fig. 2a) the pulses have a spectral width of $80 \mathrm{pm}$ and an output power of $2 \mathrm{~mW}$ and Fig. 2b) shows a pulse duration of $30 \mathrm{ps}$, which corresponds to an energy pulse of $0.74 \mathrm{~nJ}$. Fig. 2c) shows the temporary trace of the pulse train with a period of $330 \mathrm{~ns}$ between adjacent pulses to a low repetition rate of $\sim 3 \mathrm{MHz}$, so the length of the cavity is calculated to be 34.5 meters long, according to $\mathrm{f}=2 \mathrm{c} / \mathrm{n} \cdot \mathrm{L}$, where $\mathrm{n}=1.455$. To evaluate the stability and quality of the ML pulse trains, the RF spectrum is shown in Fig. 2d). The high-order harmonics yield a signal-to-noise ratio over $55 \mathrm{~dB}$ and a narrow spectral width, ensuring high stable ML operation and pulses with low timing jitter.
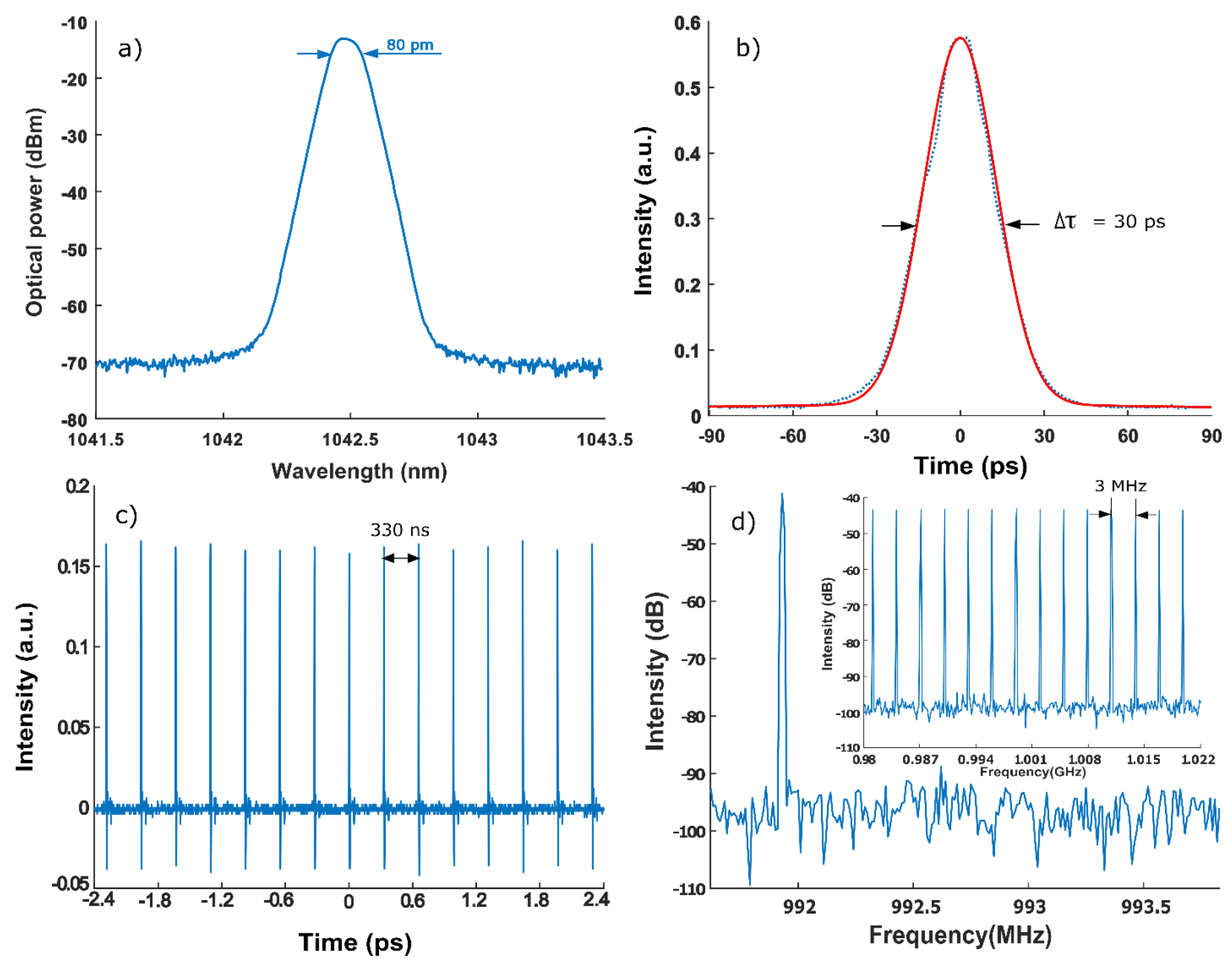

Fig.2 a) Optical spectrum, b) auto-correlation trace, c) temporal trace and d) RF spectrum measured at the YDF tunable seed laser output emitting at $1042.5 \mathrm{~nm}$ 

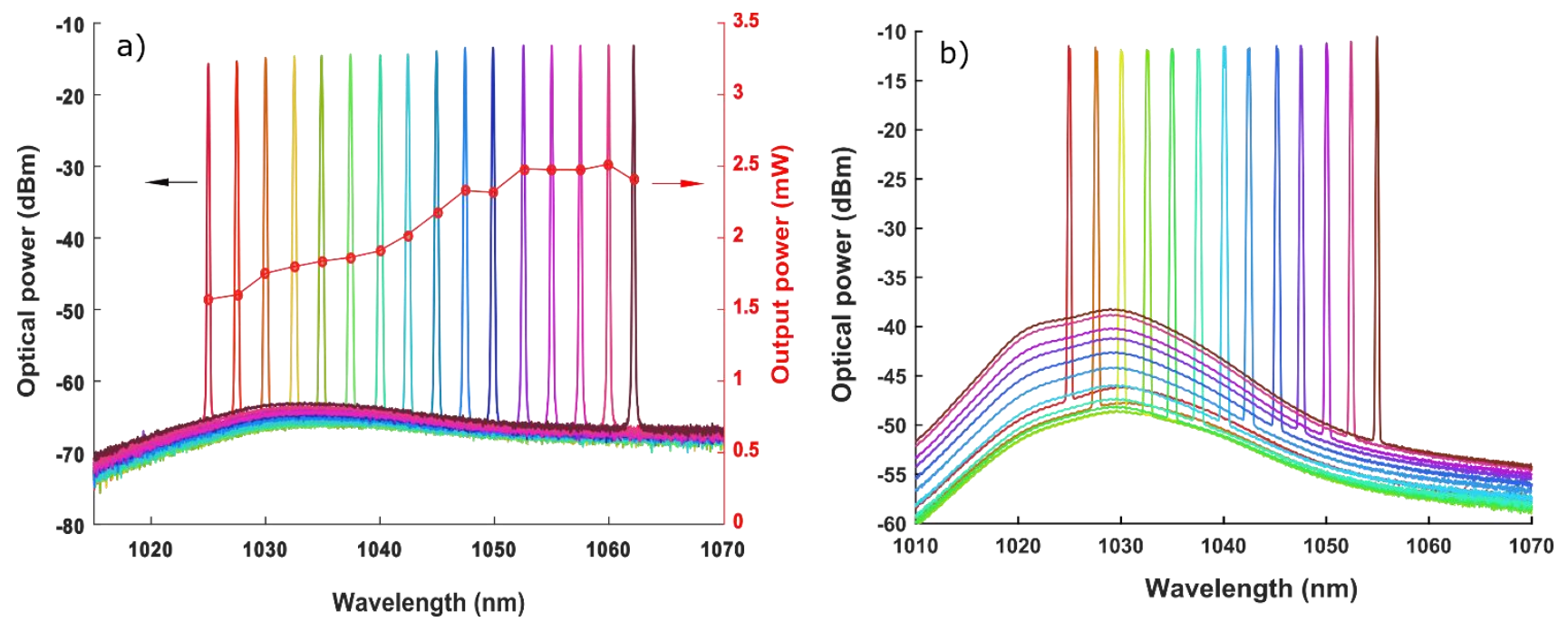

Fig.3 a) Output spectra of the tunable oscillator and the output power at each wavelength (red colored) pumping the oscillator with $78 \mathrm{~mW}$ and b) Output spectra after the amplification of the tunable oscillator.
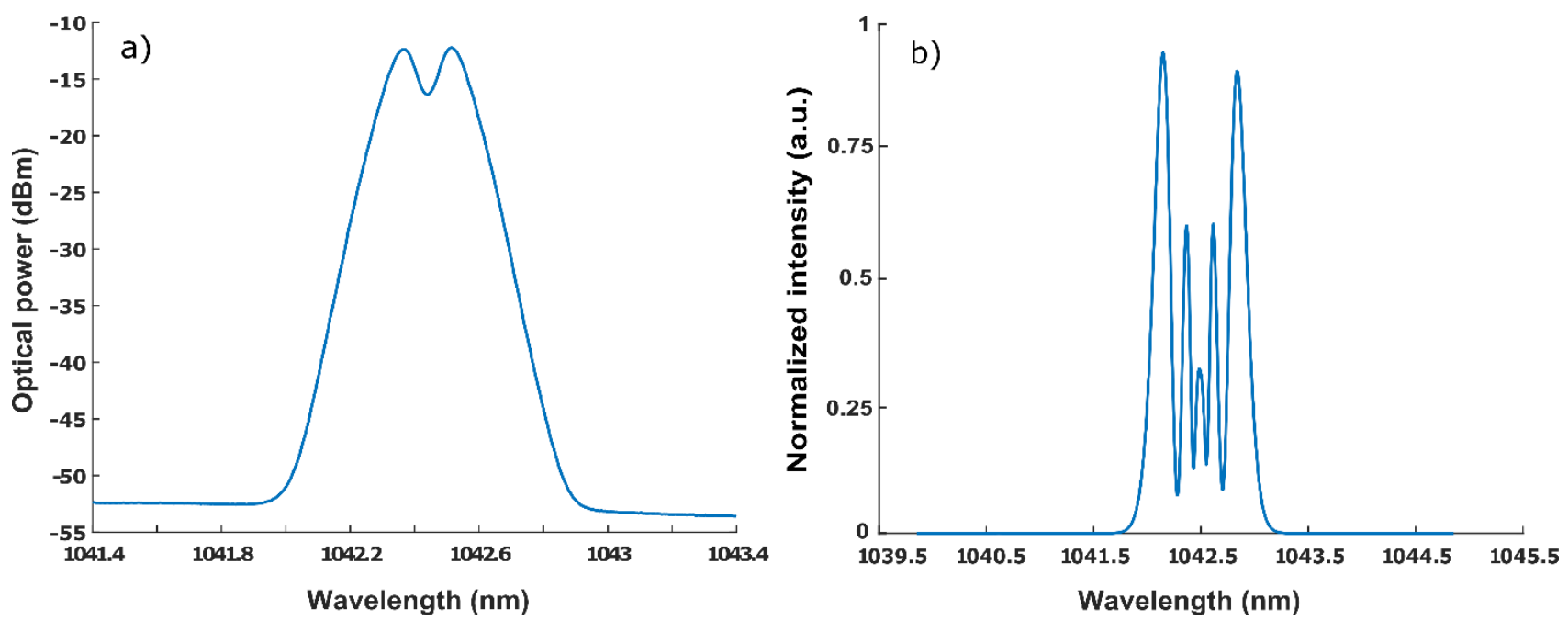

Fig.4 a) Optical spectrum of the pre-amplified tunable fiber laser and b) optical spectrum in linear scale of the tunable fiber laser (after pre and main amplification) used to pump the PCF.

This simple oscillator configuration generates a pulse train with a spectral width and a pulse duration which vary depending on the pump level from 80 to $95 \mathrm{pm}$ and from 26 to $32 \mathrm{ps}$ respectively. The continuous tunability range of the seed laser needs no polarization control and covers the spectral band from $1022 \mathrm{~nm}$ to $1062 \mathrm{~nm}$ as is illustrated in Fig.3a). The oscillator output is spliced to an isolator delivering the pulse train to the two-stage amplifier. The pre-amplifier stage is used to amplify the pulses resulting in $20 \mathrm{~mW}$ seed launched into the pulse picker (acoustic optic-modulator) to select the repetition rate from $3 \mathrm{MHz}$ to $500 \mathrm{KHz}$. This device ensures reaching the necessary peak power to drive the FWM process in the same conditions for the whole emission range of the laser, compensating the wavelength dependent variations over the whole ytterbium gain spectrum. As it has been explained, through the selection of the appropriated repetition rate, the average power reduction in part of the wavelength-emission range due to the limitations of ytterbium gain bandwidth is compensated by the incremented peak power. The resulting preamplified pulse train is launched into a main amplifier which allows to reach an average power of $\sim 400-450 \mathrm{~mW}$. This pulses reach the several $\mathrm{kW}$ peak power range, ensuring the FWM process in the PCF easily. Fig. 3b) shows the output spectra of the seed laser after main amplification. As it can be seen the tunable range has been limited from 1062 to $1055 \mathrm{~nm}$ due to the gain bandwidth restrictions of the YDF amplifiers.

In Fig.4, the spectral measurements for pre-amplified and mainamplified pulses are depicted, showing a spectral width of 0.25 $\mathrm{nm}$ and $0.8 \mathrm{~nm}$ respectively. This broadening effect is inherent to the YDF amplification process and it is caused by self-phase modulation (SPM) effect in the normal dispersion regime. This type of unwanted broadening directly affects to the bandwidth of the signals generated in the PCF by OPG and, therefore, to the generation of the CARS signal. This is because CARS generation depends on the convolution of the pump (seed laser) and the signal when these are irradiated on a biological sample and match the frequency shift of a molecular Raman resonance, so the spectral bandwidth of both mentioned signals are directly related to the resolution of the obtained CARS signal. 
To drive the parametric process of FWM, the main amplifier is spliced to the PCF using an optimized method previous alignment of the PM fibers. The correct arc fusion splice of both fibers with relative similar core diameters allows to obtain a coupling efficiency over $70 \%$, being a $73 \%$ coupling efficiency the best experimental value obtained. Fig 5a) shows the splice between YDF and PCF where the air holes of the PCF have been collapsed in a very small length. The commercial PCF LMA-5-PM of NKT Photonics utilized in this experimental setup presents its zero-dispersion wavelength at $1055 \mathrm{~nm}$ and it has been simulated using a numerical mode solver. Fig $5 \mathrm{~b}$ ) displays the calculated parametric generation of idler and signal wavelength respect to pump wavelength (blue colored) and the corresponding frequency shifts between signal and pump (red colored). In fact, the pump between $1025 \mathrm{~nm}$ and $1055 \mathrm{~nm}$ generates the signal wavelengths between 780 and $940 \mathrm{~nm}$ and allows to access resonances in the range of 1100 to $3300 \mathrm{~cm}^{-1}$. Fig.6a) shows an optical spectrum measurement of the generated signal and idler, and the residual pump after the
FWM process in the PCF. The difference between signal and pump is about $3 \mathrm{~dB}$ ensuring a high frequency conversion efficiency. The measured signal power is in the range of $23 \mathrm{~mW}$ to $42 \mathrm{~mW}$ for different pump power launched into the PCF. The output spectra of the generated signal for different pump wavelengths is depicted in Fig.6b). The signal bandwidths are in the range of 10 to $35 \mathrm{~cm}^{-1}$ FWHM.

In addition, the in-fiber band-pass filter placed into the FLM allows to control the wavelength tunability of the seed laser with a precision of $0.02 \mathrm{~nm}$ (controlling the shiftment through the band-pass filter knob). This precise control of the tunable range provides an accurate adjustment of the seed laser emission to perfectly match FMW signal generation which is crucial in order to obtain the corresponding frequency shift between signal and pump and, therefore, the required Raman resonances. Indeed, this adjustment allows to maximize the signal to noise ratio of the CARS microscopy images, providing the best contrast imaging as is depicted in Fig. 7.
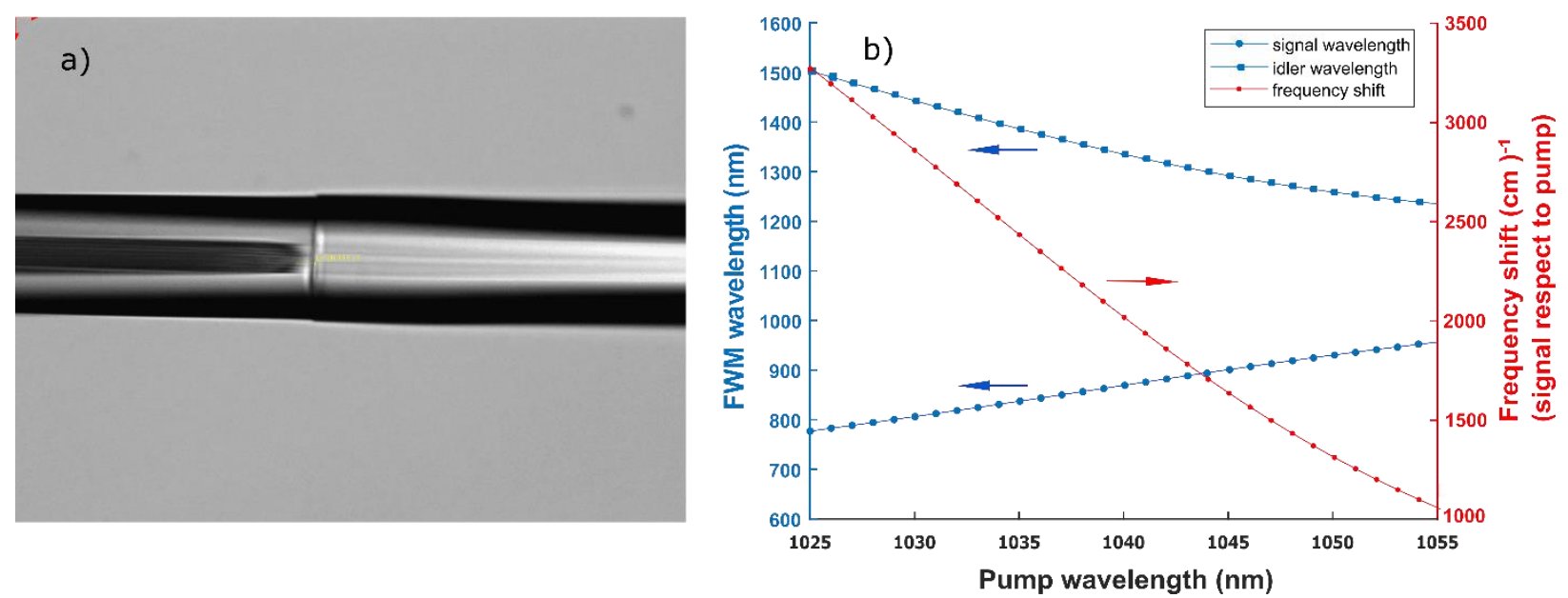

Fig.5 a) Image of the splice fusion between the PCF and the Yb-doped fiber of the main amplification stage and b) FWM phase matching diagram of the signal and idler wavelength respect to pump (blue colored) and the desired frequency shift between signal and pump wavelength (red colored).
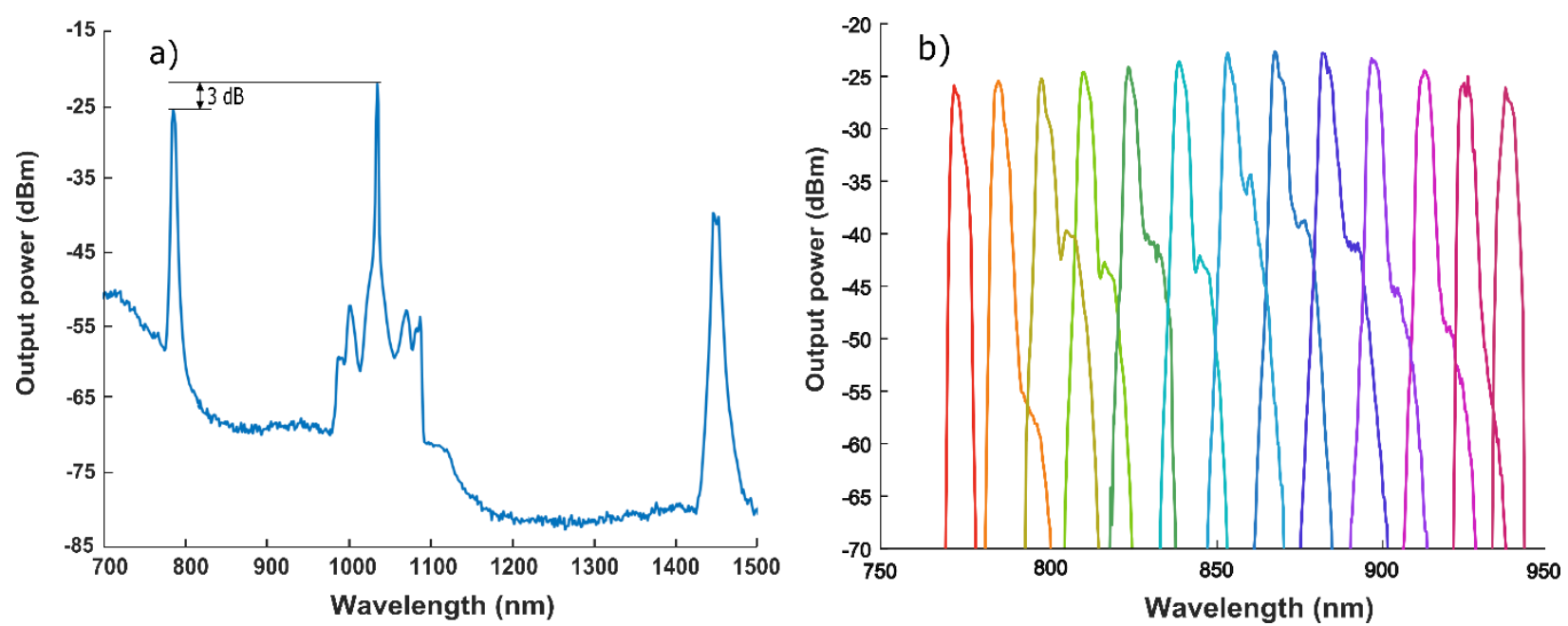

Fig.6 a) Output spectra of the idler and signal generated at $1032 \mathrm{~nm}$ pump wavelength after the FWM non-linear process in PCF and b) output spectra of the signal generated by OPG for all the tunable range. 

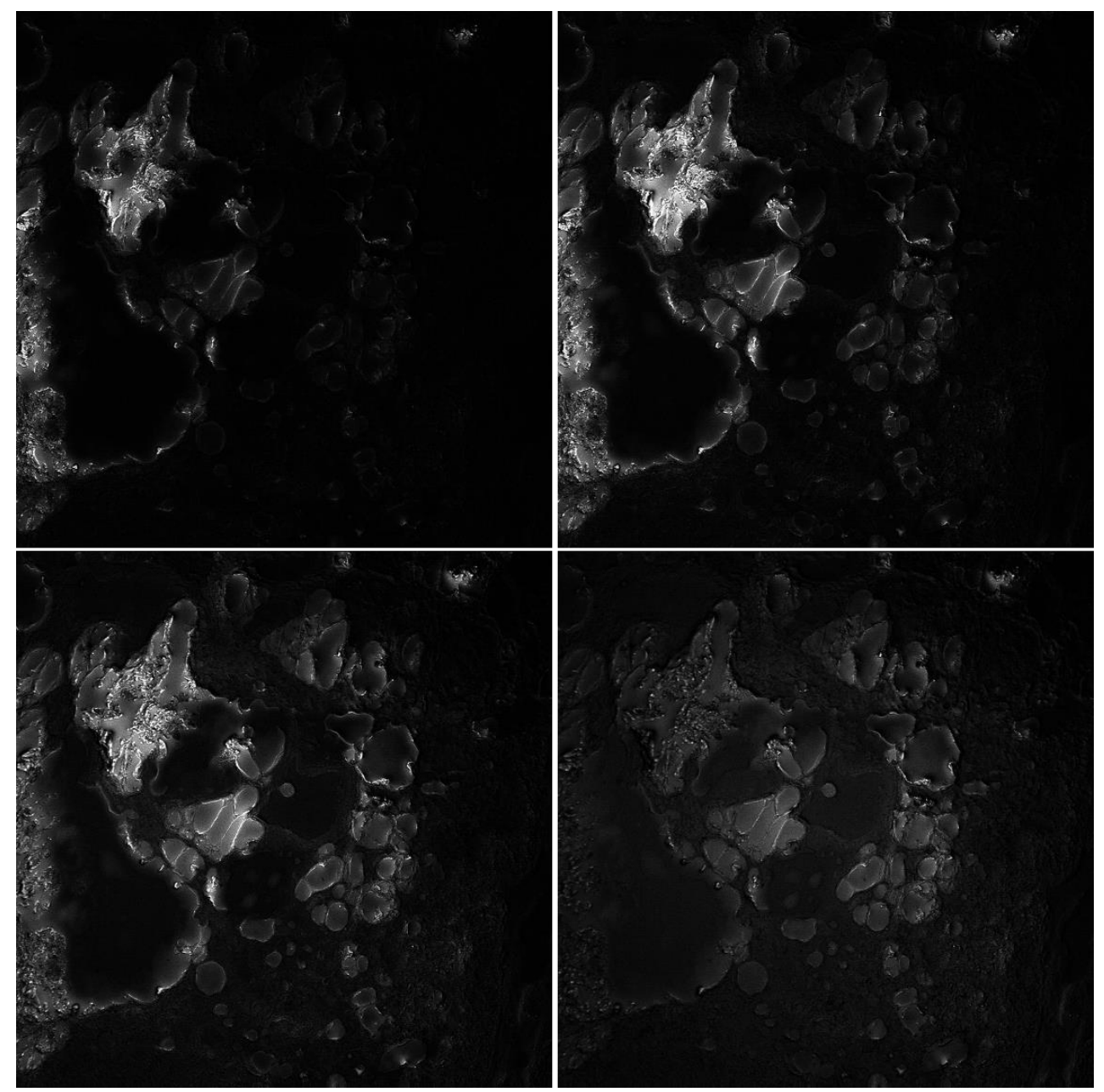

Fig. 7. CARS images showing lipid concentration measured in a human aorta with the fiber laser source reported on the manuscript.

Fig. 7 shows the obtained CARS images measuring the lipid concentration in a human aorta sample. In this image, the $\mathrm{CH}$ stretching vibration on $2850 \mathrm{~cm}^{-1}$ has been probed and the lipids are clearly visible. The difference between these images relies in the adjustment of the frequency shift between pump and signal generated in the FWM process (Stokes and pump respectively in CARS process) probing the maximum contrast (signal-to-noise ratio) with an accurate tunability of mentioned signals. As is depicted in Fig. 7, the laser source reported on this manuscript is suitable for CARS imaging application.

Finally, it could be considered to scale the repetition rate of the seed laser through an in-fiber acousto-optic modulator (AOM). This manner, the repetition rate could be reduced below $3 \mathrm{MHz}$, to increase the peak power of the pump signal and drive the FWM process with more efficiency, avoiding the need of increase the average power of the seed laser via amplification with its consequent spectral broadening caused by the SPM. Furthermore, the control of the peak power through the repetition rate could compensate the gain variations on the ytterbium region in the emission wavelengths range. This adjustment of the pump peak power in each emission wavelength would allow to reach the same conversion efficiency for the parametric process in the PCF.

\section{CONCLUSIONS}

In conclusion, we have demonstrated a broadband optical parametric generation seeded by a continuously tunable allfiber pulsed fiber laser in the near infrared for CARS imaging application with high spectral resolution and providing access to resonance between 1100 and $3300 \mathrm{~cm}^{-1}$. The complete experimental configuration is constructed of PM fibers by fusion splice yielding to alignment-free operation. The compact setup is enabled by a continuously tunable SESAM-based YDF ML seed laser with a wavelength range between 1022 to 1062 $\mathrm{nm}$, with a $3-\mathrm{dB}$ bandwidth of $-80 \mathrm{pm}$, a pulse duration of $\sim 30$ ps and a repetition rate of the pulse train of $3 \mathrm{MHz}$. After the two-stage amplifier, the pulses reach the range of several $\mathrm{kW}$ allowing the OPG based on FWM in a commercial PCF. The рump converted light via FWM generates signal and idler components in the range of 780 to $940 \mathrm{~nm}$ and 1225 to $1510 \mathrm{~nm}$ respectively. This compact, robust, reliable and low-cost structure is appealing for CARS microscopy and other life sciences applications, since it combines the pulse duration, tunability, pulse energy and spectral characteristics of a bulk $\mathrm{OPO}$ in an all-fiber integrated system. 


\section{REFERENCES}

P. Hong and Y. J. Ding, "Generation of ultra-stable signal twins and idler twins by coupled optical parametric oscillators: applications in remote sensing," in Infrared Remote Sensing and Instrumentation $X X V, 2017$, p. 104030T.

[2] A. Godard, G. Aoust, J. Armougom, E. Cadiou, D. Descloux, G. Walter, J.-B. Dherbecourt, G. Gorju, J.-M. Melkonian, and M. Raybaut, "Optical parametric sources for gas sensing applications," in Quantum Sensing and Nano Electronics and Photonics XIV, 2017, p. 101112X.

[3] T. Gottschall, T. Meyer, M. Baumgartl, C. Jauregui, M. Schmitt, J. Popp, J. Limpert, and A. Tünnermann, "Fiber-based light sources for biomedical applications of coherent anti-Stokes Raman scattering microscopy," Laser \& Photonics Reviews, vol. 9, pp. 435451, 2015.

[4] K. Yang, P. Ye, S. Zheng, J. Jiang, K. Huang, Q. Hao, and H. Zeng, "Polarization switch of four-wave mixing in a tunable fiber optical parametric oscillator," Optics express, vol. 26, pp. 2995-3003, 2018.

[5] C. Poudel and C. F. Kaminski, "Supercontinuum radiation in fluorescence microscopy and biomedical imaging applications," JOSA B, vol. 36, pp. A139-A153, 2019.

[6] J.-Y. Zhang, Optical parametric generation and amplification: Routledge, 2019.

[7] Y. Chen, Y. Li, W. Li, X. Guo, and Y. Leng, "Generation of high beam quality, high-energy and broadband tunable mid-infrared pulse from a KTA optical parametric amplifier," Optics Communications, vol. 365, pp. 7-13, 2016.

[8] A. Lombardini, V. Mytskaniuk, S. Sivankutty, E. R. Andresen, X. Chen, J. Wenger, M. Fabert, N. Joly, F. Louradour, and A. Kudlinski, "High-resolution multimodal flexible coherent Raman endoscope," Light: Science \& Applications, vol. 7, pp. 1-8, 2018.

[9] K. Yang, S. Zheng, Y. Wu, P. Ye, K. Huang, Q. Hao, and H. Zeng, "Low-repetition-rate all-fiber integrated optical parametric oscillator for coherent anti-Stokes Raman spectroscopy," Optics Express, vol. 26, pp. 17519-17528, 2018.

[10] T. Gottschall, T. Meyer, M. Schmitt, J. Popp, J. Limpert, and A. Tünnermann, "Four-wave-mixing-based optical parametric oscillator delivering energetic, tunable, chirped femtosecond pulses for non-linear biomedical applications," Optics express, vol. 23, pp. 23968-23977, 2015.

[11] I. Aporta, M. A. Quintela, and L. Higuera, "Switchable dualwavelength mode-locked fiber laser source for in-PCF parametric frequency conversion applied to CARS microscopy," Journal of Lightwave Technology, 2019.

[12] S. C. Kumar and M. Ebrahim-Zadeh, "Yb-fiber-based, highaverage-power, high-repetition-rate, picosecond source at $2.1 \mu \mathrm{m}, "$ Laser \& Photonics Reviews, vol. 10, pp. 970-977, 2016.

[13] J. Kang, X.-Y. Qi, X.-Q. Feng, L. Hou, M. Jiang, H.-W. Chen, Y. Wang, K.-L. Wang, and J.-T. Bai, "High-stability broadband wavelength-tunable single-frequency ytterbium-doped all-fiber compound ring cavity," IEEE Photonics Journal, vol. 9, pp. 1-8, 2017.

[14] H. Wu, J. Wu, Q. Yu, K. Zhang, H. Xiao, J. Leng, J. Xu, and P. Zhou, "Over $70 \mathrm{~nm}$ broadband-tunable $\mathrm{Yb}$-doped fiber pulse laser based on trilaminar graphene," Laser Physics Letters, vol. 14, p. $065105,2017$.

[15] R. J. Francis-Jones, "Photon Pair Generation via Four-Wave Mixing in Photonic Crystal Fibres," in Active Multiplexing of Spectrally Engineered Heralded Single Photons in an Integrated Fibre Architecture, ed: Springer, 2017, pp. 21-37.

[16] J.-C. Delagnes, R. Royon, J. Lhermite, G. Santarelli, H. Muñoz, T. Grosz, D. Darwich, R. Dauliat, R. Jamier, and P. Roy, "High-power widely tunable ps source in the visible light based on four wave mixing in optimized photonic crystal fibers," Optics express, vol. 26, pp. 11265-11275, 2018.

[17] D. Churin, R. Olson, N. Peyghambarian, and K. Kieu, "High power, widely tunable synchronously pumped fiber-based optical parametric oscillator," in 2016 Conference on Lasers and ElectroOptics (CLEO), 2016, pp. 1-2.

[18] M. E. Marhic, Fiber optical parametric amplifiers, oscillators and related devices: Cambridge university press, 2008.

[19] L. Zhang, J. Zhou, Z. Wang, X. Gu, and Y. Feng, "SESAM modelocked, environmentally stable, and compact dissipative soliton fiber laser," IEEE Photonics Technology Letters, vol. 26, pp. 13141316, 2014.

K. Huang, Q. Li, and H. Chen, "A switchable dual-wavelength fiber laser based on asymmetric fiber Bragg grating Fabry-Perot cavity with a SESAM," Journal of Modern Optics, vol. 63, pp. 628-631, 2016.

[21] I. A. Litago, D. Leandro, M. Á. Quintela, R. A. Pérez-Herrera, M. López-Amo, and J. M. López-Higuera, "Tunable SESAM-based mode-locked soliton fiber laser in linear cavity by axial-strain applied to an FBG," Journal of Lightwave Technology, vol. 35, pp. 5003-5009, 2017.

[22] A. F. Runge, C. Aguergaray, R. Provo, M. Erkintalo, and N. G. Broderick, "All-normal dispersion fiber lasers mode-locked with a nonlinear amplifying loop mirror," Optical Fiber Technology, vol. 20, pp. 657-665, 2014.

[23] P. K. Gupta, C. P. Singh, P. K. Mukhopadhyay, and K. S. Bindra, "Generation of stable clean ultrashort pulses in a simple all-fiber, all-normal dispersion ytterbium-doped mode-locked laser," Applied Optics, vol. 58, pp. 5533-5539, 2019.

[24] C. Kong, "Nonlinear optical microscopy based on mode-locked fiber lasers," HKU Theses Online (HKUTO), 2019.

[25] V. Lazarev, A. Krylov, D. Dvoretskiy, S. Sazonkin, A. Pnev, S. Leonov, D. Shelestov, M. Tarabrin, V. Karasik, and A. Kireev, "Stable similariton generation in an all-fiber hybrid mode-locked ring laser for frequency metrology," IEEE transactions on ultrasonics, ferroelectrics, and frequency control, vol. 63, pp. 10281033, 2016.

[26] E. U. Rafailov and E. A. Avrutin, "Q-switched and mode-locked lasers for biophotonic applications," Semiconductor Lasers and Diode-based Light Sources for Biophotonics, p. 57, 2018.

[27] J. Kang, P. Feng, X. Wei, E. Y. Lam, K. K. Tsia, and K. K. Wong, "102-nm, 44.5-MHz inertial-free swept source by mode-locked fiber laser and time stretch technique for optical coherence tomography," Optics express, vol. 26, pp. 4370-4381, 2018.

[28] P. Dupriez, "Ultrafast Fiber Lasers for Multiphoton Microscopy: High-resolution microscopy using ultrafast fiber lasers," PhotonicsViews, vol. 16, pp. 70-73, 2019.

[29] K. Kieu, "Compact ultrafast fiber lasers for multiphoton microscopy and precision measurements," in JSAP-OSA Joint Symposia, 2019, p. 20p_E214_4.

[30] M. Baumgartl, T. Gottschall, J. Abreu-Afonso, A. Díez, T. Meyer, B. Dietzek, M. Rothhardt, J. Popp, J. Limpert, and A. Tünnermann, "Alignment-free, all-spliced fiber laser source for CARS microscopy based on four-wave-mixing," Optics express, vol. 20, pp. 21010-21018, 2012.

[31] T. Gottschall, T. Meyer, M. Schmitt, J. Popp, J. Limpert, and A. Tünnermann, "High quality coherent anti-Stokes Raman scattering (CARS) microscopy imaging with an ultra-compact four-wave mixing based fiber laser sources," in Novel Techniques in Microscopy, 2015, p. NT2C. 4.

[32] Y. Li, X. Xiao, L. Kong, and C. Yang, "Fiber supercontinuum source for broadband-CARS microspectroscopy based on a dissipative soliton laser," IEEE Photonics Journal, vol. 9, pp. 1-7, 2017.

[33] J.-X. Cheng and X. S. Xie, Coherent Raman scattering microscopy: CRC press, 2016.

[34] M. Baumgartl, J. Abreu-Afonso, A. Díez, M. Rothhardt, J. Limpert, and A. Tünnermann, "Environmentally stable picosecond $\mathrm{Yb}$ fiber laser with low repetition rate," Applied Physics B, vol. 111, pp. 3943, 2013.

[35] T. Gottschall, T. Meyer, M. Baumgartl, B. Dietzek, J. Popp, J. Limpert, and A. Tünnermann, "Fiber-based optical parametric oscillator for high resolution coherent anti-Stokes Raman scattering (CARS) microscopy," Optics express, vol. 22, pp. 21921-21928, 2014.

[36] T. Bocklitz, A. Silge, H. Bae, M. Rodewald, F. B. Legesse, T. Meyer, and J. Popp, "Non-invasive Imaging Techniques: From Histology to In Vivo Imaging," in Molecular Imaging in Oncology, ed: Springer, 2020, pp. 795-812. 